\title{
Modelling Kinetic Indentation in Topocomposite Surface of Elastic Rigid-plastic Solid Bodies
}

\author{
N. Voronin \\ Department of Tribology \\ Institute of Machine Science of RAS \\ Russian Federation
}

\begin{abstract}
The mechanics of a rigid sphere contact interaction with the top composite surface of elastic rigid- plastic solids is analyzed. Analytic dependencies are obtained describing both the character of load changing on rigid pyramidal and spherical indenters versus the depth of indentation in a topcomposite and elastic and plastic properties of layered system components.
\end{abstract}

Keywords-layered system; topocomposite; thin hard coating; composite hardness; kinetic indentation

\section{INTRODUCTION}

Among known nondestructive methods of machine parts mechanical characteristics monitoring hardness tests are widely spread. It is well known a method of material micromechanical characteristics and micro hardness evaluation by examination the depth of indenter penetration under load. The method consists in continuous indenter penetration in a specimen with registration of kinetic diagram "load-depth of indentation".

Usually one uses as indenters Vickers diamond pyramids or small diameter balls made of hard alloys. The development of the methods of parts surfaces hardening by concentrated flows of energy and matter allows obtaining high-quality coatings with the thickness of a fraction of micron. Mechanical properties of such coatings may differ markedly from that of substrates [1].

It is necessary to realize that diagnostics of such surfaces by instrumental indentation method allows obtaining a design value of micro hardness, which is the complex (composited) one, and according to opinion of many researchers this value takes into account both the hardness of the coating and the substrate.

\section{ST ATE OF THE ART}

In the work [1] the mechanics of indentation of a rigid sphere of radius $R$ in elastic rigid-plastic two-layered halfspace(single-layered topocomposite) is considered. The topocompositeconsists of a top layer with thickness $h$ coherently boundedwith a substrate, which is essentially thicker than a top layer, i.e. $H>>h$. The material of the top layer has elastic characteristics- the modulus ofnormal elasticity $E_{l}$, Poisson's ratio $\mu_{l}$ and yield point (initial tensile yield stress) $\sigma_{T_{1}}$, for thesubstrate material these characteristics are $E_{0}, \mu_{0}$ и $\sigma_{T_{0}}$ corres pondingly.
In[1] a number of approximate analytic engineering dependences for the topocomposite under consideration was obtained for determination stress-strain parameters of contact and some effective characteristics (effective rig idity, effective yield point, bearing capacity and complex hardness) in terms of coating thickness, geometry of contact and physicalmechanical characteristics of topocomposite components.

In particular the following analytic dependence was obtained for the complex surface hardness of a single-layered topocomposite with hard thin coating:

$$
H_{c}=H_{0} \cdot \bar{\Phi}^{\frac{1}{2}} \cdot \Phi^{-\frac{3}{2}}
$$

Where $H_{c}, H_{0}$-complex hardness of single-layered topocomposite and substrate hardness correspondingly; $\Phi$ elastic-geometrical parameter; $\bar{\Phi}$ - limitelastic-geometrical parameter.

The eqn (1)for complex (composited) hardness allows bounding this hardness of topocomposites with known characteristics of substrate by means of some functional factors named limit elastic-geometric $\bar{\Phi}$ and current elasticgeometric $\Phi$ parameters. The mentioned parameters in analytic mode can be represented as fractionally rational functions, which terms depend on physical-mechanical characteristics of topocomposite components and on the ratio $\left(\frac{h}{a_{0}^{c r}}\right)$, where $h$ is the coating thickness and $a_{0}{ }^{c r}$ is acritical radius of the contact for a substrate. The technique obtaining of functional parameters $\Phi$ and $\bar{\Phi}$ in detail is given in [1]

Critical radius $a_{0}{ }^{c r}$ conforms to the moment of plastic deformation beginning in the substrate resulted from indention into it a rigid sphere of radius $R$. This value can be computed by formulae given in [2].

Substrate hardness, which value is used as a basic factor in eqn (1), can be determined experimentally by any of known methods. Brinell hardness $H B$ allows computing a mean pressure in the imprint formed due to a spherical indenter penetration to surface. While determining Brinell hardness it is necessary to provide conditions, under which the load on the indenter ensures maximal mean stress in the imprint. This requirement is fulfilled if the imprint diameter $d$ is with in the limits $0,2 \cdot D<d<0,6 \cdot D$, where $D$-the spherical indenter diameter. The method of Vickers hardness $(H V)$ determination 
consists in indentation of the standardized tetrahedral diamond pyramid with the square basis in a material surface under study.

Between hardness measurements by Vickers and Brinell methods there are much in common. Really at indentation of a diamond pyramid with the standardized angler $\lambda=136^{\circ}$ one receives $H V$ values close to $H B$ values obtained at an "ideal" imprint $d=0,375 \cdot D$ [3]. The depth of an imprints by Vickers method can be expressed in that case through the diameter of an imprint $d$ by Brinell one as $s=0,2 \cdot d$.

The computed hardness is directly proportional to the relation of the value of load to the sizes of an imprint and for the given material is constant value, which doesn't depend on the load (at measurement by Vickers method it is reached automatically owing to similarity of imprints irrespective of depth, whereas at measurements by Brinell method it is true for conditions $0,2 \cdot D<d<0,6 \cdot D)$.

Under kinetic indentation of a rigid sphere of radius $R$ in a rigid-plastic medium the medium is deformed first elastically and then ideally plastically. The elastic part of the diagram "load - depth of indentation" is described by the Hertz formula:

$$
P=\frac{4}{3} \cdot \frac{\sqrt{R}}{K} \cdot(t)^{\frac{3}{2}}
$$

where $t$ is the depth of indentation under spherical indenter; $K=\frac{1-\mu^{2}}{E}$.

The limiting depth of indentation $[t]$, corresponding to the transition from elastic deformation to purely plastic one, for elastic ideally plastic material is defined from the condition of equality of mean pressure in contact to the value of limiting hardness $H B=3 \sigma_{T}$ :

$$
[t]=\left(\frac{3 \pi}{2} \cdot K \cdot H B\right)^{2} \cdot R
$$

In the field of plastic deformation the dependence of load from the depth of indentation can be obtained by formula (4) transforming (see the author's paper [4]).

When indentation of rigid pyramid occurs, the field of elastic deforming is absent, and the value of micro hardness (mean pressure) at any depth of indentation can be obtained using well-known formula for Vickers hardness calculation:

$$
H V=\theta \cdot \frac{P}{s^{2}}
$$

where $\theta=0,03784$ (for $P$ in $K g f$ and $s$ in $\mathrm{mm}$ )

Experimentally obtained kineticdiagram"load - depth of indentation" allows computing not only surface hardness of the material under study but Yung's modulus and a number of other mechanical characteristics. While studying the surface hardness of hardened materials (topocomposites) it is necessary to consider effect of a substrate on the value of the composited hardness, taking into account that the character of changes of this value fro $m$ the coating thickness is unknown.

\section{PROCEDURE OF PROBLEM SOLUTION}

Let us re-arrange eqn (1), which describes layered solid theoretical hardness dependence on coating thickness, in that one describing the hardness dependence on the depth of indenter penetration $s$. For what we will consider the geometrical ratios characterizing interrelations between limit radius of aim print $a_{c}{ }^{c r}$ due to a sphere of radius $R$ indentation on the surface of a layered system with the depth of indentation of a tetrahedral pyramid.

From consideration the geometry of imprints made by pyramidal and the spherical indenters during hardness tests it is possible to receive after simple transformations the expression connecting the parameter $\frac{s_{c}}{h}$, which characterizes depth of indentation of a rigid pyramidin two-layer half-space in dimensionless form, with the parameter $\frac{h}{a_{0}^{c r}}$ characterizing coating thickness in dimensionless form as:

$$
\frac{a_{c}^{c r}}{h}=\frac{a_{0}^{c r} \sqrt{\bar{\Phi}}}{P_{0}}=0,4 \frac{s_{c}}{h} .
$$

In [1] was also shown that the hardness of topocomposites can be represented as:

$$
H_{c}=H_{0} \cdot \frac{P_{c}}{P_{0}} \cdot \frac{\delta_{0}^{c r}}{\delta_{c}^{c r}},
$$

where $P_{0}, P_{c}$ - limit loads leading to plastic deformation arising under a spherical stamp indenting in a medium with the characteristics identical withto the substrate material and in a single-layered topocomposite, $\delta_{0}^{c r}, \delta_{c}^{c r}$ - the depth of limit indentation computed for the medium with characteristics identical with substrate and for single-layered topocomposite correspondingly.

For the case of penetration of rig id (diamond) pyramidal indenter eqn (6) can be easy re-arranged in the following form:

$$
P_{c}=P_{0}^{s} \cdot \frac{P_{c}}{P_{0}} \cdot \frac{\delta_{0}^{c r}}{\delta_{c}^{c r}}=\frac{H V_{0}}{\theta} \cdot s^{2} \cdot \frac{P_{c}}{P_{0}} \cdot \frac{\delta_{0}^{c r}}{\delta_{c}^{c r}} ;
$$

Multiplying and dividing the right part of eqn (7) by " $h$ " and using eqn (1) we can write down the following analytical dependence, which connects the change of the load on the pyramid with the depth of its indentation in a topocomposite:

$$
P_{c}=\frac{H V_{0}}{\theta} \cdot h^{2} \cdot\left(\frac{s}{h}\right)^{2} \cdot\left(\bar{\Phi}\left\{\frac{s}{h}\right\}\right)^{\frac{3}{2}} \cdot\left(\Phi\left\{\frac{s}{h}\right\}\right)^{-\frac{3}{2}}
$$

Analytic expression obtained above as $P_{c}=\varphi\left(\frac{s}{h}\right)$ for one concrete type of topocomposites graphically presented in fig.1.

The curves are also presented here demonstrated the dependence "load - depth of indentation" for uniform compact 
materials with characteristics corresponding to the material of substrate $P_{0}$ and coating $P_{1}$, as well as the theoretical dependence of the surface hardness $H_{c}$ of the single-layered topocomposite change on the depth of pyramidal indenter penetration. The Figure also illustrates three typical areas of location of sites where plastic deformation orig inates. The first rangeI of the coating thicknessis characterized by the location of the point where plastic deformation originates in the coating material.

The second range II is characterized by the location of the point where plastic deformation originates in the base material and by its permanent position on the layer-coating interface within the whole range. The third range III of the coating thickness is the site where plastic deformation is located in the base material.

For the case of a rigid sphere indentation in the surface of a single-layered topocomposite the diagram "load- depth of indentation" consists of two parts. The first one belongs to the moment of elastic deformation and the second- to plastic deformation of the material under test.

For convenience of superposition of the formulae connecting the load with the indentation depth both under elastic and plastic deformations, we can represent formula (2) as:

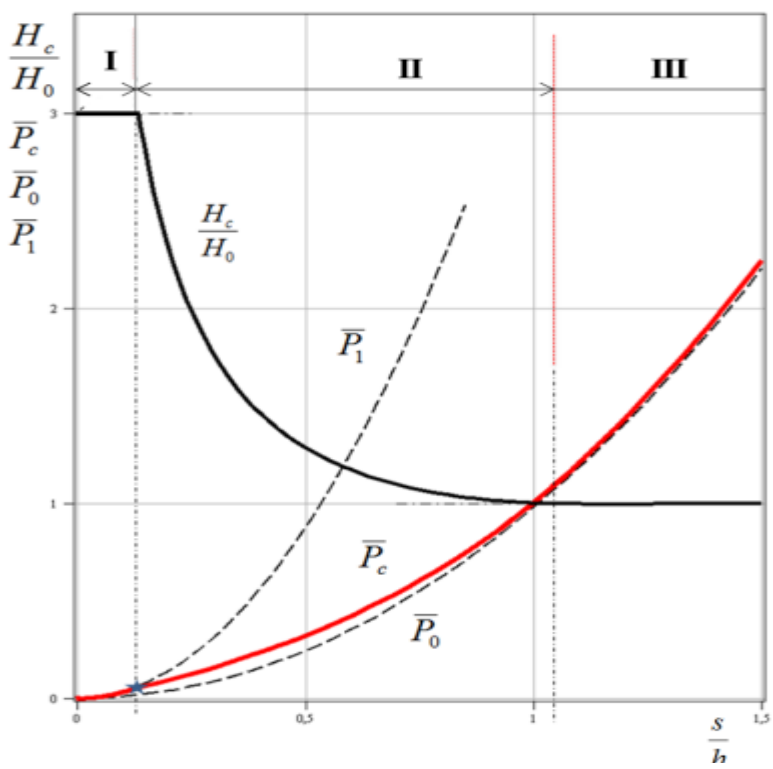

FIGURE I. DEPENDING OF THE LOADING IN TOPOCOMPOSIT E WITH PARAMETERS $\mathrm{K}=0,5$ AND $\mathrm{Y}=3$ AND IN COMPACT MATERIALS WIT HPROPERT IES OF COATING AND SUBST RATE MATERIALS, AS WELL ASTHE COMPOSITE HARDNESS OF THE TOPOCOMPOSITE VERSUS THE DEPTH OFPENETRATION BY THE VICKERS PYRAMID.

$$
P_{0}=\frac{4}{3} \cdot \frac{\sqrt{R \cdot h^{3}}}{K_{0}} \cdot\left(\frac{t}{h}\right)^{\frac{3}{2}}
$$

for the depth of indentation $0 \leq \frac{t}{h} \leq\left(\frac{3 \pi}{2} \cdot K_{0} \cdot H_{0}\right)^{2} \cdot \frac{R}{h}$.

Dependence (2) for atopocomposite would look like as:

$$
P_{c}=\frac{4}{3} \cdot \frac{\sqrt{R \cdot h^{3}}}{K_{c}} \cdot\left(\frac{t}{h}\right)^{\frac{3}{2}}
$$

for the depth of indentation $0 \leq \frac{t}{h} \leq\left(\frac{3 \pi}{2} \cdot K_{c} \cdot H_{c}\right)^{2} \cdot \frac{R}{h}$.

Or after corres ponding substitutionsit will look as:

$$
P_{c}=\frac{4}{3} \cdot \frac{\sqrt{R \cdot h^{3}}}{K_{0} \cdot \Phi^{\frac{3}{2}}} \cdot\left(\frac{t}{h}\right)^{\frac{3}{2}}
$$

for the depth of indentation

$0 \leq \frac{t}{h} \leq\left(\frac{3 \pi}{2} \cdot K_{0} \cdot H_{0} \cdot \bar{\Phi}^{\frac{1}{2}}\right)^{2} \cdot \frac{R}{h}$.

For the case of a rigid sphere indentation in a rigid-plastic solid it is possible to rewrite dependence (2) as follows:

$$
P_{c}=P_{0}^{s} \cdot \frac{P_{c}}{P_{0}} \cdot \frac{\delta_{0}^{c r}}{\delta_{c}^{c r}}=H B_{0} \cdot \pi 2 R \cdot t \cdot \frac{P_{c}}{P_{0}} \cdot \frac{\delta_{0}^{c r}}{\delta_{c}^{c r}}
$$

Multiplying and dividing the right part of expression (11) by " $h$ " and us ing early obtained expressions (1), (7) and (8) we can write down the analytic dependences connecting the load on the indenter at microhardness tests with the depth of indentation of the rigid sphere of radius $R$ :

$$
\begin{aligned}
& \text { For the depth of indentation }\left(\frac{3 \pi}{2} \cdot K_{0} H_{0} \bar{\Phi}^{\frac{1}{2}}\right)^{2} \cdot \frac{R}{h} \leq t \\
& P_{c}=H B_{0} \cdot \pi 2 R \cdot h \cdot\left(\frac{t}{h}\right) \cdot\left(\bar{\Phi}\left\{\frac{t}{h}\right\}\right)^{\frac{3}{2}} \cdot\left(\Phi\left\{\frac{t}{h}\right\}\right)^{-\frac{3}{2}}
\end{aligned}
$$

\section{CONCLUSIONS}

A transformation of the analytic expression given in [1] was proposed, which describes the dependence of complex(composited) surface hardness of a single-layered elastic rigid-plastic topocomposite on the topocomposite characteristic geometrical parameters and characteristics of spherical indenter contact with this material (coating thickness, limiting radius of elastic imprint)and physicalmechanical properties of components of layered solid (elastic constants, yield points of coating and substrate).

An analytical expression was received for complex hardness of topocomposite surface layer determination depending on the depth of penetration of rigid pyramidal and spherical indenters. Analytical expressions were also founded which allow describing the character of change of load on rigid pyramidal and spherical indenters depending on the depth of their penetration in topocomposite, elastic and plastic properties of layered system components. 


\section{ACKNOWLEDGEMENT S}

The work was done by financial support of Ministry of education and science RF, Agreement №14.607.21.0040, 22.07.2014, Project RFMEFI60714X0040.

\section{REFERENCES}

[1] Voronin, N.A., Composite and real hardnesses of thin coatings, Advanced Materials Research, 560-561, pp. 803-808, 2012.

[2] Johnson, K., Cont act Mechanics, Cambridge University Press, 1985.

[3] Bernshtein, M.L., Zaimovsky, V.A.,Mechanical properties of metals. Metallurgia, Moscow, 1979.

[4] Voronin, N.A., A theoretical model of elastoplastic penetration of the rigid sphere, Trenieilznos, 24 (1), pp.16- 26, 2003 